\title{
Cinémas
}

Revue d'études cinématographiques

Journal of Film Studies

\section{HANDLING, Piers et VÉRONNEAU, Pierre (direction). L'Horreur intérieure : les films de David Cronenberg. Paris-Montréal : Cerf - Cinémathèque québécoise, 1990, 262 p.}

\section{René Prédal}

Volume 2, numéro 2-3, printemps 1992

URI : https://id.erudit.org/iderudit/1001088ar

DOI : https://doi.org/10.7202/1001088ar

Aller au sommaire du numéro

Éditeur(s)

Cinémas

ISSN

1181-6945 (imprimé)

1705-6500 (numérique)

Découvrir la revue

Citer ce compte rendu

Prédal, R. (1992). Compte rendu de [HANDLING, Piers et VÉRONNEAU, Pierre (direction). L'Horreur intérieure : les films de David Cronenberg.

Paris-Montréal : Cerf - Cinémathèque québécoise, 1990, 262 p.] Cinémas, 2(2-3),

237-241. https://doi.org/10.7202/1001088ar d'utilisation que vous pouvez consulter en ligne. 
HANDLING, Piers et VÉRONNEAU, Pierre (direction). L'Horreur intérieure: les films de David Cronenberg. Paris Montréal: Cerf - Cinémathèque québécoise, 1990, 262 p.

David Cronenberg est aujourd'hui le plus célèbre réalisateur canadien, vivant et travaillant dans son pays. Mais anglophone et œuvrant dans le genre fantastique (au sens large du terme), il ne correspond guère à l'image "historique» que le reste du monde cinéphilique se fait du cinéma canadien («direct» et francophone). D'où le statut paradoxal de ce cinéaste qui, vu du Québec ou de France, apparaît atypique alors qu'il sera considéré à New York comme un classique metteur en scène de style posthollywoodien. Authentique auteur de films dans un créneau — le film d'horreur — qui n'en produit pas beaucoup, Cronenberg se révèle au milieu des années 70 en même temps que John Carpenter, Larry Cohen, Tobe Hooper, Steven Spielberg ou Brian de Palma ${ }^{1}$ et, au lieu de gagner sa légitimité en changeant progressivement de genre ou de s'enfoncer au contraire dans l'anonymat du film de série, il accuse sa forte personnalité en restant depuis 20 ans fidèle au même type de films. Aussi sa reconnaissance esthétique ne s'est-elle pas faite sans mal, Cronenberg n'étant au début soutenu par aucun lobby critique alors que la profession (parfois même des producteurs québécois) et les organismes fédéraux de tutelle lui étaient par contre moins hostiles.

En 1983, juste après la sortie de Videodrome, la publication de The Shape of Rage, première mouture de ce volume collectif réuni en anglais par Piers Handling, constitue donc une courageuse prise de position esthétique. L'actualisation de l'ensemble nécessitée par cette édition en français permet d'insister sur l'évolution présente du cinéaste passant de la peur viscérale au fantastique intérieur tout en conservant le même «discours sur l'homme, sur la science et sur la société» (P. Véronneau). Le cinéphile français qui ne connaît le réalisateur que depuis le festival d'Avoriaz 1983 et n'a généralement vu que ses deux derniers films sera peut-être un peu frustré par la part somme toute assez réduite du livre réservée à The Fly (1986) et Dead Ringers (1988) alors que Videodrome est analysé plusieurs fois longuement. Mais, conçu 
de manière extrêmement cohérente autour de ce qu'il convient d'appeler «l'effet Cronenberg» (P. Véronneau), ce travail collectif est finalement fort complet, juxtaposant un long entretien, une étude d'ensemble de 80 pages de W. Beard (essai synthétique et analyse film à film jusqu'en 1983) complétée par P. Véronneau pour les trois dernières œuvres, plusieurs articles consacrés à un aspect particulier de la thématique et même une vision dissidente de Robin Wood réfutée au point de vue méthodologique par John Harkness.

Il est intéressant de constater d'abord que le parcours de Cronenberg n'est pas du tout celui que l'on attendrait d'un réalisateur de films d'horreur. Au départ il se passionne pour la littérature (voulant être écrivain) puis pour la science (d'où sa mise en scène systématique de curieux savants représentant des figures parentales ou au moins professorales); son intérêt pour le cinéma ne s'affirme qu'un peu plus tard, par la fréquentation des œuvres d'auteurs européens. Ses premiers courts métrages seront donc très personnels: il les écrit, les photographie et les monte seul avant que tout à coup se conjuguent «horreur» et «public» pour établir son image de marque. W. Beard remarque fort justement que les détracteurs de Cronenberg se recrutent dans les rangs des inconditionnels de tout le cinéma d'horreur de ces 20 dernières années avec son escalade de violence et de sexualité. Aussi mène$\mathrm{t}$-il une habile défense du genre à partir de la notion de violation, montrant d'ailleurs que Cronenberg se sert du fantastique plus qu'il ne le sert puisqu'il agit en auteur complet écrivant ses propres scénarios, ce qui est fort rare dans ce type de films où règnent généralement la division du travail et les effets spéciaux (que lui-même utilise de moins en moins). De fait, «l'esprit viscéral» de son œuvre le rapproche davantage de David Lynch que de la tendance Gore.

Pour P. Véronneau, le thème fondamental de l'auteur est l'exploration des «troubles qui voient le jour dans la personnalité de quelqu'un lorsque des modifications profondes surviennent». Entre Kafka et le mythe Jekyll-Hyde, le retour d'une agressivité violente semant la destruction traduirait donc surtout le «désir de l'homme de dépasser ses limites originelles» (mythe de Faust) dans un dualisme puisé au cœur d'une dichotomie physique avant 
de déboucher dans une dialectique répression-libération. Mais pour W. Beard, délinquance, déchéance, désordre et confusion submergent finalement tout, Cronenberg parvenant souvent à concilier humour et douleur dans une espèce de Grand Guignol baroque où, comme dirait Lord Byron cité par M. Yakowar, «nous rions pour ne pas vomir».

Cronenberg joue sur les pulsions les plus secrètes, la fascination inconsciente de chaque spectateur, pour le meurtre et le sang affrontés directement dans la société d'aujourd'hui (à part The Dead Zone, tous les films sont urbains) et non avec la distanciation du passé (fantastique gothique), du futur (la science-fiction) ou de l'éloignement géographique (aventures exotiques). Cette proximité accroît le côté terrifiant de cette œuvre, passionnante parce qu'elle touche à la biologie - science «vedette» de cette fin de siècle - et plus particulièrement à la génétique qui fait la une de l'actualité scientifique: sexualité monstrueuse, mutations physiques et psychiques, transferts de personnalités, apparitions d'organes autonomes, fusion, division, gémellité, clonage rompent les équilibres naturels, provoquent folie et dégénérescence, multiplient infirmités ou troubles de comportement pour forger une «chair nouvelle» qui désintègre la nature humaine dans une sorte de pourrissement universel. L'horreur cancérigène de tumeurs proliférantes, déviations rageuses d'excroissances mortelles, verrues et embryons nauséeux peuplent une œuvre dévastatrice où les hallucinations les plus terrifiantes rejoignent les chancres physiques les plus écœurants.

Robin Wood souligne la répulsion qu'inspirent les corps affectés d'aberrations dégoûtantes et accuse le caractère malsain de toute manifestation de la sexualité (ainsi le creusement d'une sorte de vagin abdominal puis la sortie d'un appendice phallique dans Vidéodrome). Mais si Cronenberg travaille en réaction contre le laxisme des mœurs, son œuvre n'est pas pour autant réactionnaire bien qu'elle ait fréquemment été dénoncée par les groupes féministes irrités que les femmes - prédatrices ou victimes - aient rarement le beau rôle. La misogynie est donc plusieurs fois mise en question dans le volume, mais Cronenberg se défend de dispenser un message univoque: «Je ne crois pas à la simplicité des choses», «mon œuvre est ambiguë», ... duelle en tous cas 
pourrait-on affirmer en s'appuyant sur les deux films à ce jour les plus achevés, The Fly et Dead Ringers.

Au début de sa carrière, Cronenberg n'hésite pas à montrer, car ce dont il parle est justement réputé non montrable! L'ellipse ferait donc retomber la tension puisque le spectateur ne pourrait rien imaginer. Le cinéaste révèle alors le défi et filme des parasites sortant d'une bouche ou d'une épidémie de rage, bref le chaos pris dans «la fièvre nerveuse d'une histoire bien racontée» (W. Beard). Mais les deux derniers longs métrages concentrent davantage thèmes et intrigues dans des personnages très chargés: à la fois savants et sujets de leurs propres expérimentations, éléments tour à tour actifs et réflexifs. Sommet (provisoire) de cette intériorisation de l'horreur, Dead Ringers voit l'anomalie vaginale d'une comédienne provoquer la démence de deux brillants médecins dont l'équilibre gémellaire se brise sur des instruments chirurgicaux surgis d'un autre âge. P. Véronneau parle joliment de «l'émergence rougeoyante de l'anormalité» et rappelle le mot d'André Breton — «la beauté sera convulsive ou ne sera pas» - , notant que Cronenberg redéfinit quant à lui l'adage en rendant la beauté de ses films révulsive: «À travers toute mon œuvre se retrouve le thème de la transformation. Cela a à voir avec le thème de l'identité, de sa fragilité» (D. Cronenberg).

Qu'une épidémie de parasites vermiculaires se répande hors de l'immeuble contaminé (Shivers) ou que Marilyn Chambers - exreine du porno - propage une rage sanguignolante (Rabid), le lieu de ces horreurs reste Montréal où se situe également Scanners tandis que Videodrome, The Fly et Dead Ringers se déroulent à Toronto. D'où l'intérêt des textes de P. Handling et G. Pevere qui tentent de dépasser la facture américano-mondialiste inhérente au genre pour s'attacher à définir la canadianité de Cronenberg généralement occultée par la nature fantastique d'une œuvre en opposition avec la tradition réaliste et documentaire de tout l'art du pays. Mais la critique féroce d'une société à l'ordre nordaméricain, où tous les personnages sont des victimes et où la faiblesse du mâle n'a d'égale que celle de l'image du père, reflète bien le caractère d'ailleurs en grande partie insaisissable des comportements nationaux du Canada anglais. Cronenberg traduit ainsi une part refoulée tout à fait inconsciente mais réelle, suggé- 
rant que «l'image extérieure d'un Canada accommodant et pensif est un vernis qui a été entretenu uniquement au prix d'une énorme et toujours plus violente répression de ses élans psychosexuels» (G. Pevere). Nous ajouterons qu'avec ses télépathes (Scanners) ou sa télévision qui tue (Videodrome), Cronenberg appartient tout à fait au continent des technologies de la communication et plus précisément au pays de Marshall McLuhan.

David Cronenberg et Pierre Perrault offrent indiscutablement deux reflets fort différents du Canada et surtout du cinéma, mais le temps n'est plus de les opposer pour exclure l'un ou idolâtrer l'autre. L'intérêt premier de ce dossier est d'établir la dimension d'auteur d'un cinéaste qui ne fut porté ni par sa communauté culturelle (moins intéressée que le Québec à favoriser une expression cinématographique nationale) ni par le genre fantastique (où la concurrence hollywoodienne est énorme). La démonstration est convaincante et insiste également avec bonheur sur le plaisir cinématographique dégagé par des films qui savent conjuguer brillamment tous les arts du spectacle.

René Prédal

Université de Caen

\section{NOTE}

1 Dont les premiers longs métrages ne datent pas forcément de la même année mais qui s'imposent tous les cinq au public et à la critique vers 1974-1975, comme une sorte de Nouvelle Vague du H. Pictures. 